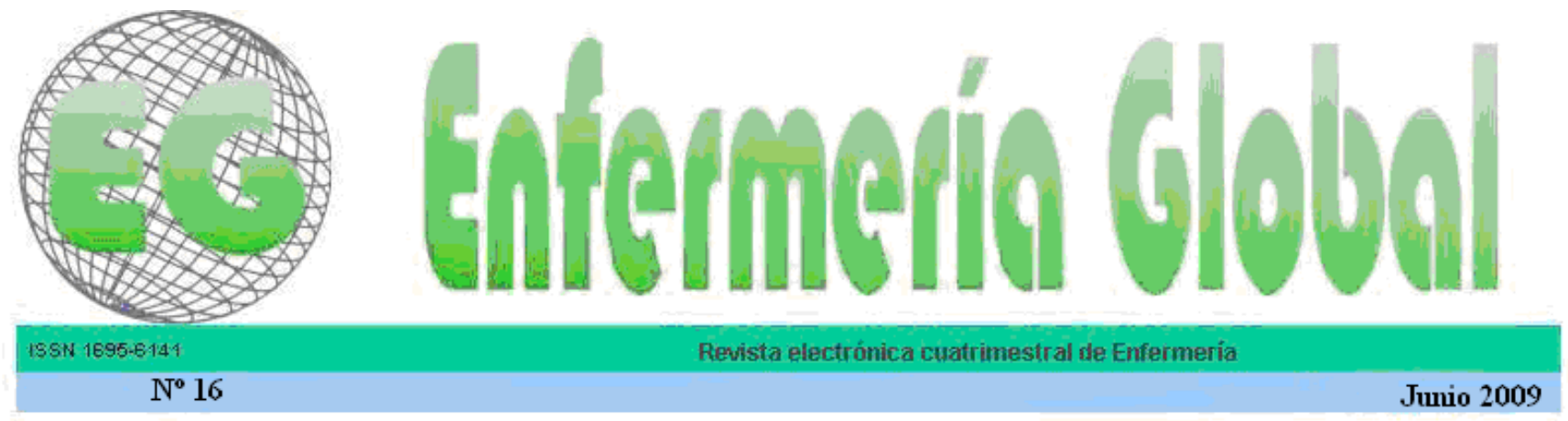

www.um.es/egloball

\title{
CLÍNIGA
}

\section{EVALUACIÓN DE LA PRESENCIA DEL SÍNDROME DE BOURNOUT EN CUIDADORES DE ANCIANOS}

\section{AVALIAÇÃO DA PRESENÇA DA SÍNDROME DE BURNOUT EM CUIDADORES DE} IDOSOS

*Da Silva, MJ, "*Braga Marques, M., " Da Silva Bruno, CT.

\begin{abstract}
*Douitora em Enfermagem. Docente. Coordenadora do Projeto A saúde do idloso: aspectos sócioculturais, político-econômicos e biológico-funcionais. **Enfermeira, integrante do Projeto A Saúde do Idoso. Departamento de Enfermagem da Universidade Federal do Ceará. Brasil.

Palabras clave: estrés psicológico; cuidadores; anciano débil

Palavras-chaves: estresse psicológico; cuidadores; idoso débil..
\end{abstract}

\section{RESUMEN}

Estudio realizado con cuidadores informales de ancianos objetivando identificar la ocurrencia del Síndrome de Burnout en cuidadores de ancianos dependientes; conocer las características socioculturales del cuidador familiar de ancianos y su percepción del cuidado como trabajo. Participaron 31 cuidadores y el instrumento fue el Maslach Burnout Inventory - MBI. De los cuidadores, un 83,6\% eran mujeres; $64,5 \%$ hijas de los ancianos cuidados. Los análisis del MBI sugieren indicios del síndrome superiores a los encontrados en profesionales de salud en los aspectos "cansancio emocional" y "despersonalización". En el aspecto "realización en el trabajo" el indicador fue satisfactorio, señalando que la relación anciano - cuidador familiar puede ser factor favorable para la no ocurrencia del síndrome por los aspectos culturales y afectivos implicados.

\section{RESUMO}

Estudo realizado com cuidadores informais de idosos objetivando identificar a ocorrência da Síndrome de Burnout em cuidadores de idosos dependentes; conhecer as características sócio-culturais do cuidador familiar de idosos e a sua percepção do cuidado como trabalho. Participaram 31 cuidadores e o instrumento foi o Maslach Burnout Inventory - MBI. Dos cuidadores, $83,6 \%$ eram mulheres; $64,5 \%$ filhas dos idosos cuidados. As análises do MBI sugerem indícios da síndrome superiores aos encontrados em profissionais de saúde nos aspectos "cansaço emocional" e "despersonalização". No aspecto "realização no trabalho" o escore foi satisfatório, indicando que a relação idoso - cuidador familiar pode ser fator favorável para a não ocorrência da síndrome pelos aspectos culturais e afetivos envolvidos. 


\section{ABSTRACT}

This study was formed with informal caregivers of elder people aiming to identify the appearance of the Burnout Disease in caregivers of dependent elder; This study also aimes to know the social-cultural characteristics of familiary caregivers of elders and their perception of the caregivers job. 31 caregivers participated in this research and the Maslach Burnout Inventory - $\mathrm{MBI}$ was the instrument used. From the caregivers, 83,6\% were women; 64,5\% were daughters of the cared elder. The analyses of the MBI suggested signs of the disease found were higher than in health professionals in the aspect of "emotional stress" and "despersonalization". In the aspect of "job achievement", the score was satisfatory, indicating the relation elder-familiar caregiver can be favorable to the non-appearance of the disease for the cultural and affective aspects involved:

\section{INTRODUCCIÓN}

La Organización Mundial de Salud -OMS- adopta en su Programa Mundial sobre el Envejecimiento nuevos paradigmas, comprendiendo que el ciclo del envejecimiento atraviesa todo el ciclo vital y, por lo tanto, impone cuidados precoces para el envejecimiento saludable. El tiempo de vida extendido puede generar necesidades de renta que, caso de no atendidas, se convertirán en problemas; el aislamiento físico y emocional podrá ser un alto factor de riesgo para la salud; la capacidad adaptativa del anciano será su arma para el buen envejecimiento ${ }^{(1)}$.

El aumento de la longevidad y con ella un aumento de la dependencia de las actividades cotidianas llevó a la OMS a reconocer la necesidad de cuidados prolongados al anciano (long-term care) definiéndolos como:

[...] el sistema de las actividades emprendidas por cuidadores informales (familia, amigos, y/o vecinos) y/o profesionales (salud, social, y otros) para asegurar que una persona que no sea capaz de autocuidarse pueda mantener la calidad de vida lo más elevada posible [.... ${ }^{(2: 6)}$

considerando las peculiaridades individuales y la preservación de la autonomía, participación, autoestima y dignidad. Para que eso sea factible se deben desarrollar políticas que garanticen la calidad de esos cuidados, tanto formales como informales, de entre los últimos, el cuidador familiar.

Entendemos como cuidador familiar una persona con lazos de consanguinidad o no con el anciano, quien asume el papel y o/tarea de cuidar de ancianos que presenten dependencias o incapacidades funcionales de tal forma que necesiten, parcial o totalmente, del auxilio de otra persona para desempeñar sus tareas cotidianas.

Esta figura, el cuidador familiar, cobra importancia cuando se observan las tendencias demográficas, políticas y comportamentales, en cuyo contexto el anciano pasa a ser una parte significativa de la población; el Estado-gobierno, dado el proceso de globalización, tiene cada vez menos capacidad de procurar políticas que atiendan a las propuestas del Estado de Bienestar, y la sociedad contemporánea coloca el hedonismo y la satisfacción personal en primer plano. Conocedor de estas tendencias el gobierno brasileño instituyó la Política Nacional del Anciano - Ley 8.842 de 1994, y el Estatuto del Anciano ${ }^{(3)}$ que destacan la permanencia del anciano en el contexto familiar como uno de los aspectos principales para mantenerlo participante, y con los cuidados cotidianos que pueda necesitar. 
El cuidado del anciano dependiente realizado por el cuidador familiar tiene aspectos positivos y negativos. En el polo positivo existe la posibilidad de un ambiente más humanizado. En el polo negativo, destacamos el desgaste del cuidador, las situaciones conflictivas entre familia, cuidador, profesionales de salud, cuyas decisiones pueden excluir al anciano de su propio cuidado, restringiendo o anulando su autonomía. En um estudio reciente, financiado por la Fundación Cearense de Amparo a la Investigación, fue posible observar algunos de estos aspectos ${ }^{(4)}$.

Desde una perspectiva sociológica, trabajo es una acción desempeñada por seres humanos, con finalidad determinada, conscientemente deseada, ejecutada mediante una participación de energía física y de inteligencia, generalmente con auxilio instrumental y que produce efecto sobre el agente. El término trabajador se adecúa al cuidador familiar, ya que "[...] todo individuo que desempeñe un trabajo creador, productivo o de transformación, que responda mínimamente a algunas necesidades humanas pasa a encuadrarse en el significado general del término". (5:1248-50)

Así, nos interesó evaluar la presencia del estrés crónico, denominado Síndrome de Burnout:

Ese síndrome se refiere a un tipo de estrés ocupacional e institucional con predilección por profesionales que mantienen una relación constante y directa con otras personas, principalmente cuando esta actividad es considerada de ayuda (médicos, enfermeros, profesores) ${ }^{(6: 1)}$.

Se caracteriza por la pérdida del interés en relación la actividad laboral, donde los eventos dejan de tener importancia y el esfuerzo personal, en el sentido de cambiar la situación, es sentido como inútil. La vulnerabilidad mayor al síndrome ocurre em los primeros años de la carrera profesional, con predominancia en mujeres, posiblemente por la doble carga de trabajo.

Algunos factores son considerados asociados al síndrome, de entre ellos la poca autonomía en el desempeño profesional; las relaciones con compañeras, clientes y autoridades, conflicto entre trabajo y familia, sentimiento de descalificación y falta de cooperación del equipo $^{(6)}$. Estos factores también están presentes en el trabajo del cuidador familiar.

El síndrome presenta como síntomas iniciales la extenuación emocional, seguida de sentimientos y actitudes negativas e insensibilidad afectiva. Por último, se manifiesta sentimiento de insatisfacción personal y en el trabajo disminución de su eficiencia y habilidad para la realización de tareas ${ }^{(6,7,8)}$.

Se han desarrollado investigaciones en esta área junto a trabajadores de salud y educadores que trabajan con personas en relación de ayuda y proximidad, donde se observan absentismo, abandono de tareas, pedido de licencias por estrés, agotamiento y depresión ${ }^{(7)}$. El síndrome constituye una de las principales enfermedades ocupacionales del mundo contemporáneo, pudiendo ocasionar desórdenes psicológicos severos en los portadores $^{(9)}$.

El interés por la temática es reciente, década de 1970, pero rápidamente se amplió por el poder explicativo de los trastornos profesionales ${ }^{(10)}$. No fue encontrada en la literatura consultada la aplicación del instrumento para cuidador no-profesional, población estudiada en esta investigación, sin embargo, estos poseen factores que también están presentes en el trabajo del cuidador profesional. 
El cuidador de ancianos actual difiere del cuidador tradicional por el aumento en la demanda por cuidados cada vez más complejos realizados en el domicilio. Sin un acompañamiento profesional, el cuidador se ve solo, desamparado, en riesgo permanente de provocar daños involuntarios al anciano cuidado por la desinformación. Este es el escenario propicio para el desarrollo del síndrome. Explorar este universo y (re)conocer sus necesidades es un paso importante para la constitución de políticas que privilegien el cuidado al cuidador. Esta investigación, aunque que de forma exploratória, puede colaborar para levantar este velo, especialmente en las actividades de atención básica de salud, donde actuamos en la docencia y en la práctica de iniciación a la investigación.

El cotidiano del cuidador familiar del anciano con dependencia puede provocar, a veces, comportamiento agresivo de ambas partes, y la sensación de exigencias excesivas por parte del anciano cuidado puede llevar al desarrollo de ese síndrome. Ya tenemos indicios de ese fenómeno en contactos con cuidadores, pero faltaba un estudio sistemático. Por la relevancia que el cuidador familiar asume en el cuidado con el anciano, necesitan ser implementadas iniciativas en los servicios de salud que recojan prevenir el estrés del cuidador. La oportunidad de este estudio está en la observación de sus resultados en el trabajo que iniciamos junto a cuidadores de ancianos con dependencia en una unidad de salud de la familia.

Los objetivos del estudio son: identificar la ocurrencia del estrés crónico en cuidadores de ancianos dependientes; conocer las características socio-culturales del cuidador familiar de ancianos y su percepción del cuidado como trabajo.

\section{MÉTODO}

Se trata de un estudio descriptivo de naturaleza cuantitativa. Fue realizada en las áreas de las Secretarías Ejecutivas Regionales -SER- I e III (Fortaleza- Ceará), comprendiendo cada una 16 barrios, en su mayoría de población de baja renta y donde son realizadas las actividades de prácticas de lãs disciplinas del Curso de Enfermería de la Universidad Federal de Ceará. En la SER I está iniciándose trabajo con cuidadores familiares en unidad de salud de la familia que tiene residencia multiprofesional en salud de la familia.

La población mayor de 60 años de Ceará representa un 7,8\% del universo populacional y la mayor de 80 años representa un $16,3 \%$ de esa población anciana, de acuerdo con el Anuario Estadístico de Ceará 2002-2003 ${ }^{(11)}$. Esto significa que hay un número elevado de ancianos con probables dependencias para la realización de sus actividades de vida diaria, necesitando de cuidador familiar.

Los sujetos de la investigación fueron cuidadores de ancianos dependientes dados de alta en las Unidades Básica de Salud de la Familia localizada en la SER III, con una población de 883 ancianos en su área de influencia y en la SER I, con aproximadamente 1.708 ancianos (11). La muestra fue seleccionada por conveniencia, a través del registro de los agentes comunitarios de salud -ACS- y el acceso a los cuidadores también se dio por intermedio de los ACS. Fueron incluidos todos los cuidadores que aceptaron participar de la investigación durante el periodo de colecta de datos en el periodo de marzo a mayo de 2005, en un total de 31 cuidadores.

Fueron aplicados dos formularios, uno conteniendo informaciones socio-culturales y del cotidiano del cuidador, cuyas informaciones fueron recolectadas por medio de entrevista estructurada con cuestiones cerradas sobre sexo del cuidador, vínculo con el anciano, estado civil, escolaridad, profesión/ocupación, renta, religión, local de residencia y 
cuestiones abiertas sobre opiniones acerca de la cualificación o no del cuidado como trabajo. Creemos que estos aspectos pueden influir en la presencia o no del estrés cronico.

La evaluación de este estrés fue mediante el Maslach Burnout Inventory - MBI, en versión en español,(12) traducida por traductor nativo de la lengua española y con adaptación de algunos términos para adecuarlo al cuidador familiar no profesional. El MBI ya fue validado por el Núcleo de Investigación Avanzadas sobre el Síndrome de Burnout del Departamento de Psicología de la Universidad Provincial de Maringá con adaptaciones de terminología direccionada a los grupos investigados(13). Este es el instrumento más utilizado en las investigaciones sobre el síndrome que llega a ser "considerado una verdadera tautología con equivalencia entre el constructo y sus instrumentos de medida, es decir el 'Burnout es lo que se mide con EI MBI (13). Está formado por una lista de preguntas y cada una debe ser respondida por medio de una escala que va desde nunca (0 puntos) hasta todos los días (6 puntos).

El formulario $\mathrm{MBI}$ evalúa los tres aspectos presentes en el síndrome: - cansancio emocional: agotamiento, extenuación emocional, fatiga, frustración, abdicación de la vida personal, estrés, todos relativos al trabajo; - despersonalización: insensibilidad, frialdad emocional, despreocupación, culpabilidad; - realización personal: comprensión, eficacia, sentimiento de actividad y de estímulo, y estado de tranquilidad La puntuación máxima, de acuerdo con el $\mathrm{MBI}$ es: cansancio emocional: 54 puntos; despersonalización: 30 puntos; realización personal: 48 puntos. Puntuación alta en los dos primeros aspectos y baja en el tercero caracterizan el síndrome. ${ }^{(12)}$

La colecta de datos por medio de visitas domiciliarias realizadas por dos estudiantes de enfermería vinculadas al proyecto de iniciación científica juntamente con los agentes de salud, estos como facilitadores de inserción de las investigadoras en las residencias de los cuidadores. El entrenamiento fue realizado por la profesora orientadora con lectura y análisis de los instrumentos de colecta. Fue realizado un pre-prueba con dos cuidadores que frecuentan la unidad básica de salud para corrección de las distorsiones y adecuación de las preguntas. Estas no formaron parte del grupo estudiado. Solamente después de las correcciones fue iniciada la colecta de datos. El local de colecta de datos fue el de conveniencia del cuidador, porque entendemos que podría haber inhibición en la presencia de otras personas, especialmente familiares.

Los datos recolectados en los dos formularios utilizados fueron organizados utilizando el software EPIINFO. Los datos socioculturales fueron organizados en tablas cruzando variables como: edad y sexo, sexo y estado civil, división de tareas y tiempo de cuidador, con uso de estadística descriptiva y analizados a partir de la frecuencia absoluta de los datos.

Destacamos que el análisis fue realizado a partir de la estadística descriptiva toda vez que la utilización de pruebas estadísticas inferenciales en una población reducida en número, como la del estudio actual, tiene bajo poder de detección de diferencias estadísticas significantes. Los datos contenidos en el $\mathrm{MBI}$ fueron agrupados según las dimensiones: cansancio emocional, despersonalización y realización personal, a continuación fueron efectuados cruces con las variables tiempo de cuidado, vínculo con el anciano y sexo del cuidador. Las opiniones sobre trabajo y tarea de cuidar fueron transcritas, por medio de hablas, componiendo el análisis de los datos.

El proyecto fue aprobado por el Comité de Ética en Investigación del Complejo Hospitalario de la UFC, conforme resolución 196/96 del Consejo Nacional de Salud. Fue presentado a 
todos los participantes el término de consentimiento libre y esclarecido y la investigación fue iniciada después de firma del cuidador.

\section{RESULTADOS}

\subsection{Caracterización del cuidador:}

Los participantes se distribuyeron entre 20 y 80 años, siendo que 12 (un 38,7\%), por ocasión de la investigación, se encontraban en la franja etaria entre 20 y 40 años, 15 (un 48,4\%) entre 41 y 59 años y 4 (un 12,9\%), entre 60 y 80 años. La muestra estuvo compuesta por 5 (un $16,1 \%$ ) hombres cuidadores y 26 (un 83,9\%) mujeres cuidadoras.

Con relación al vínculo que el cuidador tenía con el anciano cuidado, 4 (un 12,9\%) eran compañeras, 20 (un 64,5\%) eran hijos. De estos, 15 (un 75\%) eran mujeres y 5 (un 25\%) eran hombres.

Tomando en consideración el estado civil de las mujeres cuidadoras, 9 (un 34,6\%) eran casadas; 3 (un 11,5\%) divorciadas; 4 (un 15,4\%), solteras, 7 (un 26,9\%) vivían en unión consensual, y 3 (un 11,5\%) eran viudas. Los cuidadores del sexo masculino eran divorciados o solteros.

Con relación al grado de escolaridad, 5 (un 16,1\%) eran analfabetos; 20 (un 64,5\%) cursaron hasta la enseñanza fundamental y 6 (un 19,4\%) estudiaron hasta el bachillerato. De los entrevistados, 25 (un 80,6\%) no tenían trabajo remunerado. Observamos, con relación a la renta, que 14 (un 45,2\%) no tenían ninguna fuente de renta personal y el máximo encontrado fue de dos salarios mínimos. Pero tratándose de renta familiar, 12 (un 39,7\%) tenían hasta un salario mínimo, con un techo entre los entrevistados de cuatro salarios mínimos (1 cuidador).

Nos interesaba conocer la percepción del cuidador sobre la concepción de trabajo y las respuestas fueron: 2 (un 6,5\%) respondieron que era una "forma de ocupar el tiempo" , 14 (un 45,2\%), que eran "tareas que dan el sostén", 1 (un 3,2\%) respondió que era "todo de lo que se recibe pago" y 14 (un 45,2\%), que era lo que "yo hago todos los días". A los encuestados que consideraban cuidar del anciano un trabajo, 10 (un 32,3\%) respondieron que no y 21 (un 67,7\%) respondieron que sí. Las justificaciones presentadas fueron:

Porque ha muchas responsabilidades, por cuenta de la rutina (D12)

Porque es una obligación seria, da más trabajo que um niño (D7) Porque como ya tiene experiencia en cuidar de su madre, a veces es contratado (D6).

Porque pasa el día cuidando, sólo Dios podrá recompensar(D28).

Consideramos también en nuestra investigación cuánto tiempo el cuidador ejerce ese papel, y las dos franjas con mayor número fueron: hasta 5 años, 13 (un 42,0\%), y por encima de 10 años, 11 (un 35,5\%).

Las tareas de cuidar eran divididas entre el cuidador y la asistenta (1); con los nietos (18 un $58,1 \%$ ) y 11 (un $35,5 \%$ ) no contaba con nadie para ayudar a cuidar.

La franja etaria de los ancianos, objeto de los cuidados de los participantes de la investigación, es de 60 a 100 años: 12 (un 38,7\%), entre 71 y 80 años y 15 (48, un 9\%), por encima de 81 años. 


\subsection{Evaluación del síndrome de Burnout en cuidadores}

El análisis de los resultados de las dimensiones del MBI nos trajo los siguientes datos, conforme tabla I.

Tabela I: Distribuição média das dimensões da Síndrome de Burnout em cuidadores familiares de idosos dependentes quanto sexo e tempo de cuidador. Fortaleza-CE 2005.

\begin{tabular}{|c|c|c|c|c|c|c|c|}
\hline \multirow[t]{2}{*}{ Dimensões } & \multirow[t]{2}{*}{ Média } & \multirow[t]{2}{*}{$\begin{array}{l}\text { Desvio } \\
\text { Padrão } \\
\end{array}$} & \multicolumn{2}{|c|}{ Sexo (Média) } & \multicolumn{3}{|c|}{$\begin{array}{c}\text { Tempo de } \\
\text { Cuidador(Média) }\end{array}$} \\
\hline & & & Masc. & Fem. & $<1$ ano & $\begin{array}{l}1-5 \\
\text { Anos }\end{array}$ & $\begin{array}{l}\text { >de } 10 \\
\text { Anos }\end{array}$ \\
\hline Cansaço Emocional & 22,9 & 15,88 & 21,0 & 23,26 & 16,5 & 23,3 & 18,8 \\
\hline Despersonalização & 6,64 & 5,35 & 3,8 & 7,19 & 2,5 & 7,5 & 5,8 \\
\hline Realização Pessoal & 40,8 & 9,04 & 42,4 & 40,5 & 35 & 41,2 & 41,8 \\
\hline
\end{tabular}

Fonte: Dados da pesquisa.

La dimensión cansancio emocional obtuvo una media de 22,90, con valores variando entre 1,00 y 50,00, demostrando una gran dispersión de puntuación. El valor máximo de los puntos de esta dimensión es de 54 puntos. Cuando introducimos la variable sexo observamos que los valores medios son próximos, siendo 23,26 para mujeres y 21,00 para hombres. De igual manera, las medias en cuanto al tiempo de cuidador: los que cuidan hasta 5 años fue de 22,30 y los que están cuidando hace más de 10 años 30,42. El vínculo con el anciano cuidado parece influir en los indicativos de síndrome: los que son compañeros obtuvieron una media de 29,23, hijo 22,55 y sin lazos 20,75.

La segunda dimensión del instrumento, realización en el trabajo de cuidador, la puntuación máxima semejante al instrumento, 48,00(12) y la media fue 40,80 , con poca dispersión de los resultados. Esta dimensión, analizada a partir de las variables, se presentó así: en cuanto al sexo del cuidador obtuvimos 40,50 para el femenino y 42,40 para el masculino. En cuanto al tiempo de cuidador, las medias fueron: 40,30 para los que cuidan hasta 5 años y de 40,12 para los que cuidan por encima de 10 años. En la relación con vínculo con el anciano las medias fueron: compañero 42,00, hijo 40,25 y sin lazos 41,25.

En la dimensión despersonalización la puntuación máxima fue de 20 puntos mientras la del instrumento es de 30 puntos. La media fue de 6,64.

Cuando verificamos la relación con el sexo del cuidador, más mujeres se diferenciaron de los hombres siendo 7,19 y 3,80, respectivamente. El tiempo de cuidador: hasta 5 años, la media fue de 6,76, y de más de 10 años fue 7,71. Con relación al vínculo con el anciano cuidado, los resultados de las medias fueron: compañero, 10,0; hijo 6,8; y sin lazos 3,2, Podemos inferir que hay un riesgo mayor para el compañero cuidador, y lo sin lazos con el anciano presentan poco potencial para la despersonalización.

\section{DISCUSIÓN}

La discusión sobre lo que es trabajo junto a los entrevistados indicó que, en gran número (un $67,7 \%$ ), los cuidadores ven lo que hacen como trabajo, lo que se relaciona con la perspectiva sociológica del término ${ }^{(5)}$. Em cuanto al perfil de los cuidadores, la mayoría de los entrevistados, un $83,9 \%$ eran mujeres, y se encontraban en la franja de la media edad, 
entre 41 y 59 años (un 48,4\%); un 77,4\% eran compañeros o hijos; un 34,6\% eran casados; un $83,9 \%$ residían con el anciano cuidado; un $64,5 \%$ sólo estudiaron hasta la enseñanza fundamental y un $80,6 \%$ sin renta personal. Este perfil corresponde a estudios realizados en el país y en otros lugares. ${ }^{(14)}$

Destaca que la cuidadora mujer, media edad, como es el caso de la mayoría de nuestra investigación está entrando em el proceso de envejecimiento más evidente, la menopausia, con toda la carga física y psicológica que el fenómeno acarrea. Estas pasan a asumir la tarea de cuidar de los padres o cónyuges más ancianos además de las responsabilidades de la casa y familia que ya desempeñan.

Fue observado que casi todos los cuidadores eran practicantes de alguna religión (30), concordando con la literatura ${ }^{(15)}$, que destaca la búsqueda de la espiritualidad como medio de enfrentamiento, contribuyendo a superar sentimientos negativos y a encontrar sentido a la tarea.

El sentido de cuidar como parte de la obligación familiar, referido por varios de los entrevistados, parece atenuar el desgaste provocado por la tarea de cuidar. La literatura confirma que las relaciones familiares son relevantes en el sentido de abarcar relaciones de reciprocidad, afecto, gratitud, pero también rabia y resentimiento ${ }^{(15)}$.

En estudios realizados con médicos ${ }^{(16)}$ y equipo de enfermería ${ }^{(17)}$ para evaluación del Síndrome de Burnout, podemos observar que hubo semejanza con nuestros hallazgos. Para los médicos del estudio ( $\mathrm{N}=144)$, la media del aspecto cansancio emocional fue de 22,61 y para el equipo de enfermería $(\mathrm{N}=228)$ fue de 19,43 . Los cuidadores de la investigación en foco tuvo media de 22,90, superior a los dos grupos ${ }^{(16,17)}$. Este dato puede ser indicativo que los cuidadores familiares viven en un contexto de dificultades financieras, acumulación con las tareas domésticas, cuidados con la familia y aun consigo propios, pudiendo originar situaciones de estrés. En algunos casos, lo que percibimos durante la colecta de datos fue que muchos cuidadores olvidan cuidar de sí mismos, dando siempre más importancia al estado de salud del anciano cuidado.

Durante la entrevista, 13 (un 41,9\%) participantes relataron que el agotamiento emocional varía conforme el estado del anciano, a veces un deterioro del cuadro clínico del anciano, confirma el aumento de este cansancio a lo largo del tiempo en las mujeres cuidadoras, hijas o compañeras, conforme datos de la investigación. Cuidar no es una situación lineal en que son vivenciados siempre los mismos sentimientos, pues existen conflictos $y$ ambivalências. $^{(18)}$

En el aspecto despersonalización, la media de nuestro estudio fue de 6,64, superior al estudio referido sobre los médicos(16) - 6.03, y sobre el equipo de enfermería(17) - 5,62. Los efectos negativos del cuidado para el cuidador familiar son enumerados en varios trabajos y citan el estrés, fatiga, pérdida del tiempo libre, pérdida de la privacidad, coste financiero, pérdida del sueño, ansiedad (14). Se observa que el MBI utiliza la expresión tratar "como objeto" la persona cuidada lo que causa impacto y negación, pero, en el conjunto de los ítems evaluados la puntuación máxima estuvo en los ítems "endurecimiento emocional" y culpabilidad "del cuidador por el anciano".

Este aspecto -despersonalización - es el más crítico para analizar el síndrome por tener un número reducido de cuestiones e ir de frente a la naturaleza de la actividad de cuidar. Pero es relevante destacar que el fenómeno es influenciado por el tiempo de cuidado y el vínculo del cuidador con el anciano. Las mujeres y las compañeras son las más vulnerables a la 
despersonalización. Este puede ser un mecanismo de defensa contra el sufrimiento prolongado de ver a un ser querido debilitándose pero, por otro lado, acarrea una culpabilidad muy intensa. Una de las entrevistadas lloró al relatar que:

"una vez maltraté mi madre con palabras pues estaba en mi límite humano", pero después dijo: "me arrepentí amargamente".(D15)

Este es un suceso esperado, pues [...]

En algunas ocasiones, las tensiones y dificultades derivadas de la situación del cuidado de un familiar anciano pueden conducir al surgimiento de rencores, conflictos y resentimientos entre las personas que participan de la situación. En otros casos, la dificultad derivada de la situación de cuidado se hace agravada por la presencia de sentimientos negativos y relaciones ajadas entre los miembros de la família ${ }^{(18) .}$

Por último, el aspecto realización en el trabajo evaluado por el $\mathrm{MBI}$ fue el de mayor escore medio, 40,80, superior al del personal de enfermería ${ }^{(17),}$ cuya media de satisfacción fue 38,25 , pero inferior a los médicos ${ }^{(16)}$ del estudio, que tienen media de 41,38 . No hubo variación significativa em cuanto a las variables analizadas. El cuidado, además de los factores negativos, también tiene beneficios, como el sentimiento de cumplir con el deber y responsabilidad parental, retribuir el cuidado recibido cuando niño, sentirse bien con el deber cumplido, hacer el bien y otros ${ }^{(17)}$. Los lazos afectivos, si por un lado amplían la ansiedad frente a las tareas de cuidar y la desesperanza de no ver resultados visibles en lo que hace. El anciano tiende, con el paso del tiempo, a agravar el cuadro. Por otro lado, el cuidar proporciona unejercicio de amor, de afecto, de donación de sí, que tiene un componente de fe en el ser humano, en la ética humana, de la trascendencia del Yo. Por lo tanto, la búsqueda de la esencia del ser humano.

\section{CONCLUSIONES}

Los cuidadores familiares de ancianos dependientes estudiados se asemejan a las características consagradas en la literatura: la mayoría es mujer, hija, sin renta propia, baja escolaridad y residente en la misma casa del anciano. Entienden el cuidado como un trabajo por las responsabilidades asumidas. Los cuidadores estudiados presentan indicativos del Síndrome de Burnout, principalmente en los aspectos "cansancio emocional" y despersonalización". En estos, los escores son superiores a los encontrados en profesionales de salud. En el aspecto "realización en el trabajo" presentó positividad, indicando que el cuidado prestado por familiares tiene factores positivos como el afecto, la donación de sí, la fe y la trascendencia, aspectos que no están presentes en el cuidado profesional, cuyos vínculos se limitan a los aspectos morales y profesionales de cada uno. Es importante el estudio más profundizado de la problemática del cuidador familiar y lo que este cuidado acarrea en la vida de cada uno.

\section{REFERENCIAS BIBLIOGRÁFICAS}

1.Organização Pan-americana de Saúde. Saúde dos idosos. Envelhecimento e saúde: um novo paradigma. In: 50 a Sessão do Comitê Regional. 25a Conferência Sanitária Pan-americana. Organização Pan-americana de Saúde/ Organização Mundial de Saúde. 1998 set 21-25; Washington (D.C): OPAS/OMS; 1998. p. 20. 
2. World Health Organization. Ageing and health programme. Towards an International Consensus on polity for long-term-care of the ageing. Geneva: WHO: Milibank Menorial Fund; 2000.

3. Tapai GMB, coordenador. Estatuto do idoso e legislação correlata. São Paulo: Revista dos Tribunais; 2004.

4. Moraes GLA, Silva MJ. Explorando o universo do cuidado de idosos dependentes pelo cuidador familiar. Rev RENE , Fortaleza (CE) 2004 jan-jul; 5 (1): 33-40.

5. Maravell J.M. Trabalho. In: Silva B, coordenador. Dicionário de Ciências Sociais. Rio de Janeiro(RJ): Fundação Getúlio Vargas; 1986. p. 1248-50.

6. Ballone GJ. Síndrome de Burnout. Psiqweb Psiquiatria Geral, 2002. Disponível em: http://www.psiqweb.med.br/cursos/stress4.html . Acessado em: 22 fev 2006.

7. Albaladejo R, Villanueva R, Ortega $P$, Astasio $P$, Dominguez MEC. Síndrome de Burnout em el personal de enfermería de un hospital de Madrid. Rev Esp Salud Pública, jul-ago 2004; 78(4): 505-16.

8.Vianey EL, Brasileiro ME. Saúde do trabalhador:condições de trabalho do pessoal de enfermagem em hospital psiquiátrico. Rev Bras Enfermagem, Brasília (DF) 2003 set-out: 56(5):555-557.

9 Associação Médica do Paraná. Estresse: cuidado! Você pode ser vítima de um apagão. Revista Medicina\&Cia, no 10. Disponível em: http//www.amp.org.br/noticias7/r10estresse.htm. Acessado em: 29 nov 2005.

10. Murofule MT, Abrantes SS, Napoleão AA. Reflexões sobre estresse e burnout e a relação com a enfermagem. Rev Latino-am enfermagem, São Paulo 2005 mar-abr; 13(2): 255-61.

11. Instituto de Planejamento do Ceará. Anuário estatístico do Ceará 2003-2004. Disponível em: http://www.iplance.ce.gov.br/publicaçoes/anuario 2004/tabelas.htm. Acessado em: 7 set 2005.

12. Confederación General del Trabajo. España: Guía de salud laboral. España. Disponível em: http://www.fesibac.com/publicaciones/saludlaboral/Guias/gsl19.pdf. Acessado em: 12 ago 2004.

13. Benevides-Pereira ANT. Burnout: o processo de adoecer pelo trabalho. In: BenevidesPereira ANT, organizadora. Burnout: quando o trabalho ameaça o bem-estar do trabalhador. São Paulo: Casa do Psicólogo; 2002. p. 21-91.

14. Abbott $M$, Koopman-Boyden $P$. Cuidadores informales de las personas mayores dependiente: ¿Quiénes son? ¿Qué hacen? ¿Qué les sucede? Rev Psiquiatria 1997;14(3):11120.

15. Néri AL, Carvalho VAML. O Bem-estar do cuidador: aspectos psicossociais. In: Freitas EV, Py L, Neri AL, Cançado FAX, Gorzoni ML, Rocha SM. Tratado de geriatria e gerontologia. Rio de Janeiro (RJ): Guanabara Koogan; 2002. p. 778-88.

16. Benevides-Pereira ANT. O adoecer dos que se dedicam à cura das doenças. O Burnout em um grupo de médicos. In: Benevides-Pereira ANT, organizadora. Burnout: quando o trabalho ameaça o bem-estar do trabalhador. São Paulo (SP): Casa do Psicólogo; 2002. p. 105-32. 
117. Benevides-Pereira ANT. As atividades de enfermagem em hospital: um fator de vulnerabilidade ao burnout.. In: Benevides-Pereira ANT, organizadora. Burnout: quando o trabalho ameaça o bem-estar do trabalhador. São Paulo (SP): Casa do Psicólogo; 2002. p. 13356.

18. Universidad Autónoma de Madrid [página en línea].España: Cuidar a los que cuidan. Sobre los cuidadores. Disponível em: http://www.uam.es/centros/psicologia/paginas/cuidadores/.htm. Acessado em: 22 jun 2002. 